\title{
Fetomaternal Outcome of Pyrexia in Pregnancy: A Prospective Study
}

\author{
Jhuma Biswas $^{1}$, Kaushik Banerjee ${ }^{2}$, Poushali Sanyal ${ }^{3 *}$, Mousumi Datta ${ }^{2}$, Subhendu Choudhury ${ }^{3}$, Shyamal Dasgupta ${ }^{4}$, \\ Sayantanee Sen Gupta ${ }^{3}$, Amar Nath Dey ${ }^{1}$
}

\begin{abstract}
Objectives: Fever in pregnancy is a common clinical problem which increases risk of morbidity of the mother and fetus. We studied the variable medical complications of pregnant women suffering from fever and the possible fetal complications.

Materials and Methods: In this research 183 pregnant women with fever were studied prospectively. Necessary investigations to detect the underlying cause were performed. Patients with fever due to septic abortions and blood transfusions were excluded. Maternal complications and adverse perinatal outcomes in terms of preterm delivery, perinatal death, low birth weight (LBW) and low Apgar score at 5 minutes after birth were recorded. Frequency of occurrence of maternal complications was compared according to their age, parity and period of gestation.

Results: Malaria was found to be the leading cause of pyrexia among pregnant mothers (35\%). Both maternal and fetal complications were highest when malaria was the cause of fever. Low birth weight was the most common adverse pregnancy outcome and there were 4 perinatal deaths. No statistical association was present between age, parity or period of gestation and frequency of occurrence of maternal complications in antepartum febrile illness.

Conclusion: Our study depicts that a wide range of maternal medical complications as well as fetal and neonatal complications occur due to pyrexia in pregnancy from various etiologies.

Keywords: Low birth weight, Pregnancy, Pregnancy complications, Pregnancy outcome, Pyrexia
\end{abstract}

\section{Introduction}

Fever in pregnancy is a common clinical problem worldwide. The risk to the mother and fetus is significantly increased in pregnancy complicated by infection and fever. Obstetricians often face problems in treating pyrexia in pregnancy due to its atypical presentations. Maternal immune function is usually decreased in normal pregnancy and many of the potent antibiotics should be used with caution in pregnant women due to the risk of teratogenicity $(1,2)$. Therefore, some febrile diseases may take a more severe course in pregnancy leading to transplacental transmission of infectious agents and fetal jeopardy. Further, intrapartum fever in absence of infection has also been found to be associated with increased risk of developing neonatal hypoxic encephalopathy and unexplained neonatal seizures $(3,4)$. In presence of maternal fever fetus is exposed to various inflammatory mediators as measured by umbilical cord blood cytokines even in documented absence of neonatal sepsis (5).

The underlying maternal cytokine polymorphism is strongly associated with both intrapartum fever and cerebral palsy at term $(6,7)$. Studies have shown that increased brain temperature increases oxygen consumption lower- ing the threshold of hypoxic injury. Hypothermia ameliorates hypoxic brain injury in term neonates $(8,9)$. So it remains doubtful whether hyperthermia per se, independent from the underlying infection causes neonatal brain injury or they act together.

Here we have performed a study on pregnant women suffering from fever of infectious origin to detect the various life threatening medical complications leading to severe maternal morbidity and its impact on fetal outcome. The specific objectives of our study were to assess the highly variable medical complications of the pregnant women suffering from fever according to the etiological basis and also the possible fetal complications.

\section{Materials and Methods}

Present study was a prospective observational study conducted in the Department of Obstetrics and Gynecology, Eden Hospital, Medical College and Hospital, Kolkata. All prenatal mothers admitted with fever in the ward between June 2010 and May 2011 were eligible to participate in the study. Patients with septic abortions and pyrexia due to transfusion reaction and having hypertension, diabetes, renal and cardiovascular disease were excluded. Total 183

\section{Received 2 October 2014, Accepted 4 April 2015, Available online 1 July 2015}

${ }^{1}$ Bankura Sammilani Medical College and Hospital, Bankura, West Bengal, India. ${ }^{2}$ Kolkata Medical College and Hospital, Kolkata, West Bengal, India. ${ }^{3}$ Institute of Postgraduate Medical Education \& Research, Kolkata, West Bengal, India. ${ }^{4}$ North Bengal Medical College and Hospital, Darjeeling, India.

*Corresponding author: Poushali Sanyal, Institute of Postgraduate Medical Education \& Research, Kolkata, West Bengal, India. Tel: +919830279680, Email: poushali.sanyal@yahoo.co.in 
subjects were recruited in the study. After having obtained a written informed consent from the selected patients, a detailed clinical history including their base line data comprising of age, parity and the trimester of pregnancy at diagnosis of fever was recorded and a physical examination was made. All the patients were subjected to blood investigations like hemoglobin level, complete blood count, thin and thick peripheral blood smears and malarial antigen detection (10). We did Widal test to rule out enteric fever in the second week of pyrexia and liver function test for liver disorders wherever indicated. Viral hepatitis was diagnosed by IgM capture Elisa technique. We also performed a routine microscopic examination and culture sensitivity of urine for all patients and sputum smear microscopy for fever with cough of more than 2 weeks duration for diagnosis of tuberculosis (TB). All investigations were performed maintaining standard procedures. The subjects were followed up from enrollment in study till discharge from hospital after delivery. All incidences of maternal complications and perinatal outcome in terms of preterm delivery (delivery before 37 completed weeks of gestation), perinatal death (fetal or newborn death from 28 weeks of gestation to 7 days of age), low birth weight (LBW) baby $(<2.5 \mathrm{~kg})$ and poor Apgar score (Apgar score $<7$ at 5 minutes after birth) were recorded. All personal identifier, clinical and laboratory data was entered in a semi-structured predesigned case record form for individual subjects. Microsoft Excel 2010 was used for calculation of frequencies and percentages. Statistical software StatCal was used for statistical tests of significance.

\section{Results}

We studied 183 pregnant women with fever. Different etiologies of fever were malaria (35\%), TB (3.8\%), urinary tract infections (UTIs) $(21.3 \%)$, respiratory tract infections (RTIs) (16.4\%), viral hepatitis (10.4\%), chicken pox (6\%) and typhoid (7.1\%). Table 1 shows the demographic profile of the study population. Majority of the patients were within the age bracket of 20-30 years, presented with fever in their second trimester of pregnancy and were nullipara (parity recorded at the time of diagnosis of fever). Percentage of maternal medical complication in each demographic stratum was calculated.
Table 1. Distribution of Medical Complications According to Demographic Profile of the Study Population $(n=183)$

\begin{tabular}{lccc}
\hline Demographics & $\begin{array}{c}\text { No. of } \\
\text { Cases (\%) }\end{array}$ & $\begin{array}{c}\text { Medical } \\
\text { Complications (\%) }\end{array}$ & P-Value $^{\text {a }}$ \\
\hline Age group (y) & $32(17.4)$ & $11(34.4)$ & .8 \\
$<19$ & $120(65.5)$ & $35(29.1)$ & \\
$20-30$ & $31(16.9)$ & $8(25.8)$ & \\
$>30$ & & & \\
Gestational period & $69(37.7)$ & $25(36.2)$ & .5 \\
First trimester & $103(56.2)$ & $27(26.2)$ & \\
Second trimester & $11(6.01)$ & $4(36.3)$ & \\
Third trimester & & & \\
Parity & $116(63.4)$ & $38(32.7)$ & \\
Nullipara & $67(36.6)$ & $18(26.8)$ & \\
Parity $\geq 1$ & &
\end{tabular}

${ }^{a} P<.05$ is significant. There is no statistical association between demographic characteristics and maternal complications as no $P$-value is significant.

We applied chi-square test for detecting association between incidence of maternal complication and demographic categories. No statistical association was present between demographic characteristics and maternal complications. Distribution of maternal complications and adverse fetal outcome according to specific etiology of fever is shown in Table 2. Maximum cases of fever were due to malaria followed by UTI.

Complications encountered in the study were pleural effusion, pneumonia, severe anemia, diarrhea, hypoglycemia, convulsion, jaundice and acute renal failure (ARF). Overall jaundice was the most common complication (19 cases). Case count was also high for diarrhea and hypoglycemia. Complications were most frequent when malaria was the cause of fever. Proportion of complication was also high for typhoid and hepatitis. Anemia was associated with other complications. We only considered the cases of severe anemia as major complication. There were total 14 cases of severe anemia. Six cases of severe anemia were not associated with any other complication. Figure 1 shows cause specific distribution of the complications. Here the data is single response, only the most important compli-

Table 2. Distribution of Maternal Complications and Adverse Fetal Outcomes According to Etiology of Fever

\begin{tabular}{|c|c|c|c|c|c|c|c|}
\hline \multirow{2}{*}{ Etiology } & \multirow{2}{*}{$\begin{array}{l}\text { Maternal Medical } \\
\text { Complications (\%) }\end{array}$} & \multicolumn{6}{|c|}{ Fetal Outcome (\%) } \\
\hline & & LBW & Preterm & IUGR & $<7$ Apgar & Perinatal Death & Total Adverse Outcome \\
\hline Malaria, $n=64$ & $37(57.8)$ & $50(48.1)$ & $32(48.5)$ & $30(54.5)$ & $47(57.3)$ & $3(75)$ & $55(85.9)$ \\
\hline $\mathrm{TB}, \mathrm{n}=7$ & $2(28.5)$ & $5(4.8)$ & $1(1.5)$ & $5(9.1)$ & $5(6.1)$ & - & $6(85.7)$ \\
\hline UTI, $\mathrm{n}=39$ & - & $28(26.9)$ & $20(30.3)$ & $8(14.5)$ & $17(20.7)$ & - & $29(74.3)$ \\
\hline RTI, $n=30$ & $6(20)$ & $8(7.7)$ & $2(3.0)$ & $6(10.9)$ & $4(4.9)$ & - & $11(36.6)$ \\
\hline Viral hepatitis, $n=19$ & $9(47.3)$ & $3(2.9)$ & $4(6.1)$ & - & $2(2.4)$ & - & $4(21)$ \\
\hline Chicken pox, $\mathrm{n}=11$ & $2(18.8)$ & $7(6.7)$ & $4(6.1)$ & $6(10.9)$ & $7(8.5)$ & $1(25)$ & $8(72.7)$ \\
\hline Typhoid, $n=13$ & $6(46.1)$ & $3(2.9)$ & $3(4.5)$ & - & - & - & $3(23)$ \\
\hline Total (\%), n = 183 & $62(33.8)$ & $104(56.8)$ & $66(36)$ & $55(30)$ & $82(44.8)$ & $4(2.1)$ & $116(63.3)$ \\
\hline
\end{tabular}

Abbreviations: TB, tuberculosis; UTI, urinary tract infection; URI, respiratory tract infections; LBW, low birth weight; IUGR, intrauterine growth retardation. 


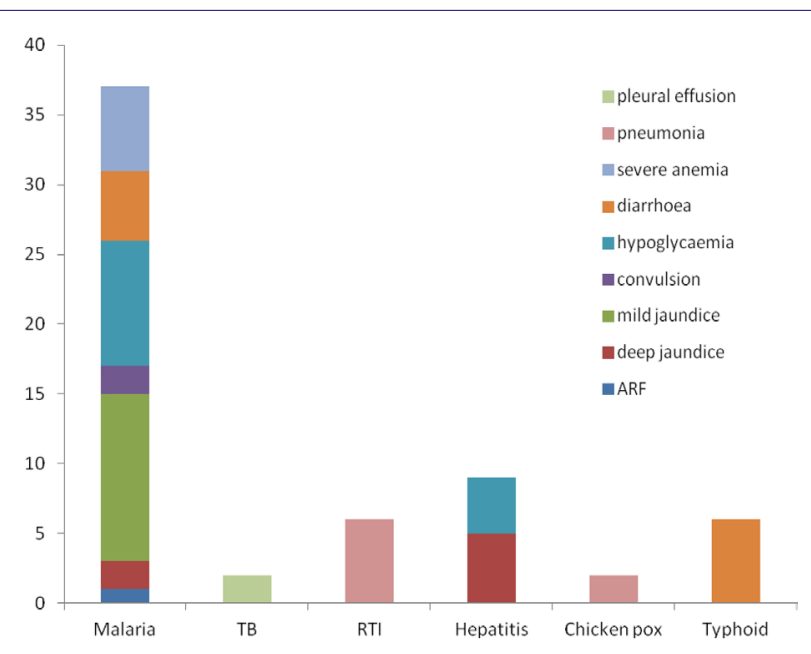

Figure 1. Component Bar Diagram Showing Different Medical Complications According to Etiology of Fever $(n=62)$.

cation is considered. Adverse fetal outcomes like LBW, intrauterine growth retardation (IUGR), preterm delivery, Apgar score $<7$ at 5 minutes after birth and perinatal mortality were studied for these pregnancies with antepartum febrile illness. All deliveries were singleton and the mean birth weight was $2.44 \mathrm{~kg}(\mathrm{SD}=0.41)$. More than half of the babies were of LBW, there were 4 perinatal deaths and overall $63 \%$ deliveries had at least one or more than one adverse outcome.

Distribution of specific etiologies of fever for each type of adverse fetal outcome was also calculated. LBW babies were the most common adverse event. Malaria in pregnancy contributed to the maximum proportion of cases for all types of adverse outcome. None of the cases of UTI in pregnancy presented with maternal complication but there was considerable adverse fetal outcome with UTI. There were few cases of TB and chicken pox but most cases had an adverse pregnancy outcome.

\section{Discussion}

During pregnancy the fetal body temperature is mainly controlled by uteroplacental circulation and heat exchange at the amniotic fluid interface. These limited routs of heat transmission from the mother maintain fetal temperature approximately $0.5^{\circ} \mathrm{C}$ to $0.75^{\circ} \mathrm{C}$ higher than that of its mother (11). Effects of pyrexia on pregnancy depend on the extent of temperature rise. Minimal temperature rise during preimplantation period and severe exposures during embryonic and fetal development might result in miscarriage, preterm birth, intrauterine growth restriction and still birth. Morishima et al, utilizing radiant heat to produce maternal hyperthermia of $107^{\circ} \mathrm{F}$ in anesthetized baboons, observed increased uterine activity and deterioration of fetal condition (12). Similarly, Cefalo and Hellegers demonstrated fetal jeopardy in anesthetized gravid ewes with the level of hyperthermia that produced maternal cardiovascular collapse (13). In our study rather than miscarriage commonly occurring fetal complications included preterm birth, IUGR, LBW, fetal demise and poor Apgar score (less than 7) at 5 minutes after birth. There were newborns with more than one complication. A study conducted by Lieberman et al (4) showed a strong association between intrapartum fever and low Apgar score, increased requirement of resuscitation and neonatal seizures in the first 24 hours following birth. Probably compromised circulation resulting from infection related vasculitis and higher metabolic demands in febrile condition might play a role in earlier development of fetal acidosis. Other than perinatal morbidity we have tried to find out various atypical clinical presentations of pregnant mothers arising in febrile condition. Previously three studies reported that nulliparous mothers were more likely to exhibit intrapartum fever than parous women (15-17). One possible explanation might be the increased metabolic expenditures leading to contractions of uterine and skeletal musculature in nulliparous mothers as compared with parous mothers (18).

Our study focused on fever in the antenatal period. The majority of our study subjects were nulliparous. Hyperthermia, typical effect of infectious agents, inflammatory reaction compounded by maternal complication has profound effect on the fetus. While maternal complications were very much dependent on the etiology of fever, fetal outcomes overlapped irrespective of the cause of fever. Moreover, adverse fetal outcome was more numerous than maternal complication. Hence it can be hypothesized that hyperthermia related changes in the uterine environment can affect fetal well-being.

\section{Conclusion}

A wide range of maternal medical complications as well as fetal and neonatal complications occur due to pyrexia in pregnancy from various etiologies that range from preventable infections like malaria to hepatitis. Hence standard methods for infection control in homes, communities and health care settings should be emphasized.

\section{Ethical issues}

Ethical clearance was obtained from the institutional ethics committee of Medical College, Kolkata, where the study was performed.

\section{Financial Support}

None declared.

\section{Conflict of Interests}

None declared.

\section{Acknowledgments}

The authors wish to thank Professor Partha Sarathi Chakraborty, Head, Department of Obstetrics and Gynaecology, Medical College and Hospital, for the support given during the compilation of this study.

\section{References}

1. Rolbin SH, Morningstrar BA. The febrile parturient. Textbook of obstetric anesthesia. New York: Churchill Livingstone; 1999:375-391. 
2. Gibbs RS, Sweet RL. Maternal and fetal infections. Maternal-fetal medicine. Principles and practice. Philadelphia: WB Saunders; 1989:656-725.

3. Impey L, Greenwood C, Mac Quillan K, Reynolds M, Sheil O. Fever in labour and neonatal encephalopathy: a prospective cohort study. BJOG. 2001;108(6):594597. doi:10.1016/s0306-5456(00)00145-5.

4. Lieberman E, Lang J, Richardson DK, et al. Intrapartum fever and neonatal outcome. Pediatrics. 2000;105(1):8-13. doi:10.1542/peds.105.1.8.

5. Goetzl L, Evans T, Rivers J, Suresh MS, Lieberman E. Elevated maternal and fetal serum interleukin-6 levels are associated with epidural fever. Am J Obstet Gynecol. 2002;187(4):834-838. doi:10.1067/ mob.2002.127135.

6. Chaiworapongsa T, Romero R, Kim JC, et al. Evidence for fetal involvement in the pathologic process of clinical chorioamnionitis. Am J Obstet Gynecol. 2002;186(6):1178-1182. doi:10.1067/ mob.2002.124042.

7. Simhan HN, Krohn MA, Zeevi A, Daftary A, Harger G, Caritis SN. Tumor necrosis factor- $\alpha$ promoter gene polymorphism -308 and chorioamnionitis. Obstet Gynecol. 2003; 102(1):162-166.

8. Yager J, Towfighi J, Vannucci RC. Influence of mild hypothermia on hypoxic ischemic brain damage in the immature rat. Pediatr Res. 1993;34(4):525-529.

9. Gluckman PD, Wyatt J, Azzopardi D, et al. Selective head cooling with mild systemic hypothermia after neonatal encephalopathy: multicentre randomized trial. Lancet. 2005;365(9460):663-70. doi:10.1016/ s0140-6736(05)17946-x

10. Levinson W, Jawetz E. Medical microbiology and immunology. 4th ed. Noewalk, Conn: Appleton and Lenge; 1999:274-276.

11. Walker DW, Wood C. Temperature relationship of the mother and fetus during labor. Am J Obstet Gynecol. 1970;107(1):83-87.

12. Morishima HO, Glaser B, Niemann WH, James LS. Increased uterine activity and fetal deterioration during maternal hyperthermia. Am J Obstet Gynecol. 1975;121(4):531-538.

13. Cefalo RC, Hellegers AE. The effects maternal hyperthermia on maternal and fetal cardiovascular and respiratory function. Am J Obstet Gynecol. 1978;131(6):687-694.

14. Petrova A, Demissie K, Rhoads GG, Smulian JC, Marcella S, Ananth CV. Association of maternal fever during labor with neonatal and infant morbidity and mortality. Obstet Gynecol. 2001;98(1):20-27.

15. Herbst A, Wolner-Hanssen $\mathrm{P}$, Ingemarsson I. Risk factors for fever in labor. Obstet Gynecol. 1995;8695):790-794.

16. Alexander JM, McIntire DD, Leveno KJ. Chorioamnionitis and the prognosis for term infants. Obstet Gynecol. 1999;94(2):274-278.

17. Sturchler D, Menegoz F, Daling J. Reproductive history and intrapartum fever. Gynecol Obstet Invest. 1986;21(4):182-186. doi:10.1159/000298951.

18. Marx GF, Loew DA. Tympanic temperature during labor and parturition. Br J Anaesth. 1975 ;47(5):600602. doi:10.1093/bja/47.5.600.

Copyright ( 2015 The Author(s); This is an open-access article distributed under the terms of the Creative Commons Attribution License (http://creativecommons.org/licenses/by/4.0), which permits unrestricted use, distribution, and reproduction in any medium, provided the original work is properly cited. 